\title{
PHARMACOPHORIC SCREENING OF VARIOUS ENDOPHYTIC FUNGAL METABOLITES
}

\author{
BRINDHA DEVI P ${ }^{1 *}$, RAJAGOPAL K², ESTHER ELIZABETH ${ }^{2}$
}

${ }^{1}$ Department of Bio-Engineering, School of Engineering, Vels University, Chennai, Tamil Nadu, India. ${ }^{2}$ Department of Biotechnology, School of Life Sciences, Vels University, Chennai, Tamil Nadu, India. Email: pbrindhadevi@gmail.com

Received: 18 January 2017, Received and Accepted: 11 February 2017

ABSTRACT

Objective: To screen various endophytic fungal metabolites toward anti-inflammatory, anticancer, and antioxidant property virtually.

Methods: In this study, 14 bioactive compounds reported from endophytic fungi have taken for structure-based drug design. With the help of software Schrodinger, different modules were used to perform screening of top active compounds. Ligprep, epharm, Glide, Quikprop are the modules were used from the software for our study. Identification of leads, pharmacophore model generation, and molecular docking studies were assessed using this software.

Results: After the screening of molecules virtually, the most bioactive anti-inflammatory compound was found to be cycloepoxytriol with the docking score of $-7.511 \mathrm{kcal} / \mathrm{mole}$, and the most active anticancer compound was found to be Phomol with the docking score of $-9.778 \mathrm{kcal} / \mathrm{mole}$. The most active antioxidant compound was found to be Phomol with the docking score of $-9.970 \mathrm{kcal} / \mathrm{mole}$. Further account the potential of the compounds to act as efficient drug candidates, their absorption, distribution, metabolism, and excretion properties were also predicted. All the compounds were shown to correlate well with all properties virtually.

Conclusion: In conclusion, using structure-based drug design, we have obtained some promising leads for anti-inflammatory, anticancer, and antioxidant drug discovery. The discovery of compounds from natural products is very potent for formulating new drugs.

Keywords: Endophytes, Anti-inflammatory, Anticancer, Antioxidant, Absorption; distribution; metabolism and excretion.

(C) 2017 The Authors. Published by Innovare Academic Sciences Pvt Ltd. This is an open access article under the CC BY license (http://creativecommons. org/licenses/by/4. 0/) DOI: http://dx.doi.org/10.22159/ajpcr.2017.v10i5.17157

\section{INTRODUCTION}

Endophytic fungi are suggested to have played a major role in structuring plant communities and in shaping processes such as colonization, competition, coexistence, and soil nutrient dynamics. Endophytic fungal diversity is shaped by environmental or habitat conditions in which the plants take residence [1]. The search of a bioactive compound from isolated endophytic fungi and higher plants are considered for developing the therapeutic drugs. Thus, there is a need to isolate and synthesize the antibiotics and therapeutic from endophytic fungi, which are highly effective, low toxic and having minor environmental impact. The discovery of new and novel anti-inflammatory drugs is an area of intense interest in both pharmaceutical industry and academic laboratories [2]. Significant advances have been made in the treatment of inflammatory diseases such as rheumatoid arthritis and multiple sclerosis, but most dramatically with new biologic agents. Natural products and their derivatives represent more than $50 \%$ of all the drugs in clinical use worldwide. Higher plants contribute not less than $25 \%$ of the total [15]. Almost $60 \%$ of drugs approved for cancer treatment are of natural origin. A wide range of natural compounds has been reported for their antiproliferative and apoptosis triggering strategies in cancer cells. Reactive oxygen species, such as $\mathrm{O}^{2-}, \mathrm{H}_{2} \mathrm{O}_{2}$, and $-\mathrm{OH}$, are generated byproducts of biological reactions, which can cause oxidative damage to biomolecules and play key roles in programmed cell death and stress-response signaling in conjunction with antioxidant production $[3,16]$. This study investigates endophytic fungi with anti-inflammatory, anticancer, and antioxidant activities to explore the potential sources of novel natural antibiotics. The preliminary screening of various endophytes for anti-inflammatory, anticancer, and antioxidant was developed for this study.

\section{METHODS}

Design of novel inhibitors using structure-based drug design Computational details

The computational works were carried out in Windows 7 operating system with the intel I5 processor with the memory of 4 GB RAM.
The desired modules Glide, Phase, LigPrep, and Quik prop were implemented with Maestro Software package (Schrodinger, LLC) 2016 to perform our in-silico analysis. The bonding orders and charges were added to the hetero atoms, and the hydrogen atoms were added to all the atoms in the utilized protein molecule [4]. Furthermore, the water molecules have been removed to prevent from additional binding.

\section{Protein preparation using protein preparation wizard}

Initially, the protein preparation was performed using protein preparation wizard, and the energy minimization of the protein file was done. About 500 cycles of steepest descent (SD) and 5000 cycles of conjugate gradient methods with optimized potential for liquid simulations (OPLS) 2005 force field using Schrodinger suite version 9.3. Grid: A rectangular box surrounding the active site was located, and the grid file was generated using receptor grid generation panel. The "Write extra precision (XP) descriptor information" option was selected and "compute RMSD" option was enabled and rest of the parameters were kept as default [4]. The input RMSD of the crystal ligand was also calculated with the prepared ligand and the original ligand.

\section{Development of energy-based pharmacophore models}

Energy-based pharmacophore was employed for finding the bioactive component of ligands against the receptor was employed. The pharmacophore sites were generated using the PHASE module of the Schrodinger. For generation of e-pharmacophore docking post processing module option was selected and the input file has to be given in.xpdes format [4]. A default set of ten chemical features: Hydrogen bond acceptor (A), hydrogen bond donor (D), hydrophobic $(H)$, negative ionisable $(\mathrm{N})$, positive ionisable $(\mathrm{P})$, and aromatic ring (R) features were used. Hydrogen bond acceptor sites were represented as vectors along the hydrogen bond axis in accordance with the hybridization of the acceptor atom. Hydrogen bond donors were represented as projected points, located at the corresponding hydrogen bond acceptor positions in the binding site. Projected points allowed the possibility for structurally dissimilar active compounds 
to form hydrogen bonds to the same location, regardless of their point of origin and directionality. Each pharmacophore feature site was first assigned an energetic value equal to the sum of the Glide XP contributions of the atoms comprising the site, allowing sites. This allowed sites to be quantified and ranked on the basis of these energetic terms $[4,5]$.

\section{Preparation of ligands}

The selected 14 endophytic bioactive molecules were processed through redundancy checking and Lipinski filters to select compounds that had better drug property (Fig. 1). All the structures were prepared using LigPrep (LigPrep v2.2, Schrodinger LLC, New York, NY) along with Epik (Epik v1.6, Schrodinger, LLC, New York, NY) to expand protonation and tautomeric states at $7.0 \pm 2.0 \mathrm{pH}$ units. Conformational sampling was also performed for all database molecules using the ConfGen search algorithm. Confgen with OPLS 2005 force field was applied for the generation of conformers with duplicate poses eliminate if the RMSD was $<2.0 \AA$. A distance-dependent dielectric constant of 4 and maximum relative energy difference of $10 \mathrm{kcal} / \mathrm{mol}$ were applied. Using phase, the database was indexed with automatic creation of pharmacophore sites for each conformer to allow rapid database alignments and screening.

\section{Molecular docking}

The predicted pharmacophore models were utilized to screen the 14 bioactive compounds [1,2]. Molecular docking for the selected compounds was carried out by glide (grid based ligand docking with energetics). The glide module comes with the package of three modes of docking, high-throughput virtual screening (HTVS), standard precision (SP), and XP mode. The XP mode was used for exhaustive sampling and advanced scoring, resulting in even higher enrichment $[5,6]$. Final shortlisting of hit molecules were performed based on visual inspection of important amino acid interactions in the active site cavity, docking scores and the hydrogen bonds involved in binding $[7,8]$.

Absorption, distribution, metabolism, and excretion (ADME) prediction

The selected 14 bioactive compounds were subjected to ADME prediction analysis using QikProp a module of Schrödinger. Various properties like LogP (octanol-water coefficient), \% human oral absorption, Lipinski's rule of five, QPlogBB (Predicted brain/blood partition coefficient), QPlogHERG (predicted inhibitory concentration values for blockage of HERG $\mathrm{K}^{+}$QPPCaco (predicted apparent Caco-2 cell permeability in $\mathrm{nm} / \mathrm{s}$ ) were predicted for the compounds and were checked for any violations [9].

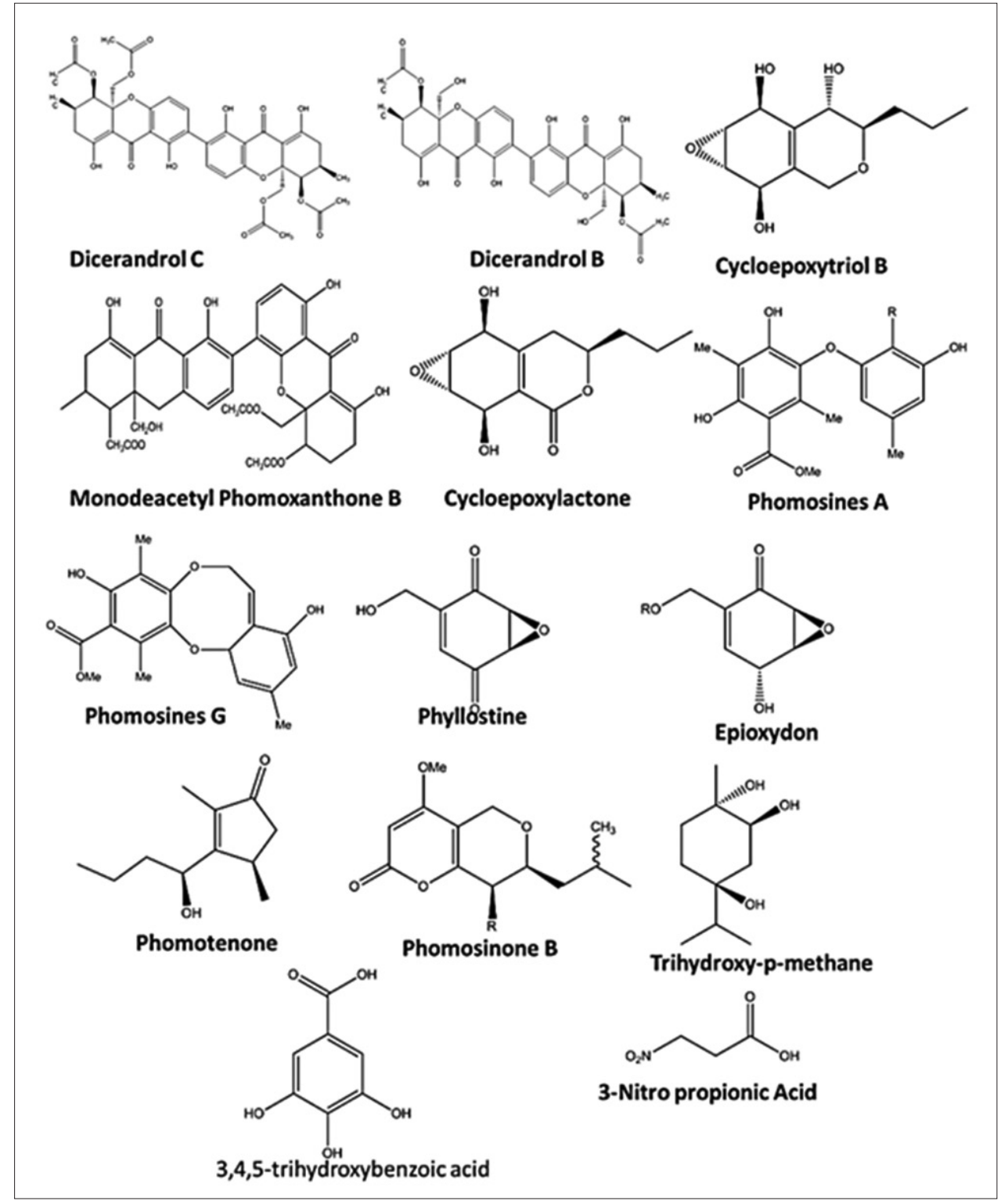

Fig. 1: List of compounds taken for the study 


\section{RESULTS}

Developing novel endophytic bioactive compounds is one of the most promising drug targets for anti-inflammatory, anticancer, and antioxidant drug discovery. Structure-based drug designing was employed for screening of bioactive compounds. In this study, we have taken three different crystal structure (PDB ID: 4PH9, 4JSR and 5FVP) for screening of anti-inflammatory, anticancer, and antioxidant class of inhibitors. We have used HTVS, SP, and XP docking options for virtual screening of molecule [9]. Glide, phase and quickprop modules utilized for docking, screening and pharmacokinetic prediction with well-defined physicsbased scoring terms and thorough with sampling of database ligands.

\section{Protein preparation and active site validation}

The respective proteins for all the three targets were retrieved from the protein data bank, and the proteins were prepared using protein preparation wizard. The hydrogen atoms were added, water molecules were removed and the minimization of energy was done by converging the heavy atoms using OPLS_2005 force field. After protein preparation, grid: A rectangular box surrounding the active site pocket were validated, and the corresponding spaces were identified. The interaction of different ligands and the protein in the active site pocket were also identified $[10,11]$.

\section{Anti-inflammatory, anticancer, antioxidant enzyme target docking} study

We attempted new drug design strategy, which was not explored for this target; we started with 3 inhibitor/substrate (ibuprofen, Sirt-3, and nitric oxide) bound with protein being crucial in the identification of novel lead bioactive molecules. The crystal ligand was made to docked into their respective proteins, and the docking score was found to be $-9.75 \mathrm{kcal} / \mathrm{mol},-11.73 \mathrm{kcal} / \mathrm{mol}$ and $-6.637 \mathrm{kcal} / \mathrm{mole}$, respectively, with the important amino acid interaction like (Arg121 and Try356), (Phe157, Glu296, Ile230, Arg158, Asp231 and Val292) and (Glu592 and Asn569). The three diagrammatic representation of crystal ligand in the active site of protein and its ligand interaction diagram are shown in Fig. 2a-c, respectively. After reference ligand docking, superimposition was calculated using original pose ligands and the docked poses ligands in the active site of the protein.

The RMSD were found to be $<2$ and the diagrammatic representation were depicted in Fig. 3a-c respectively.
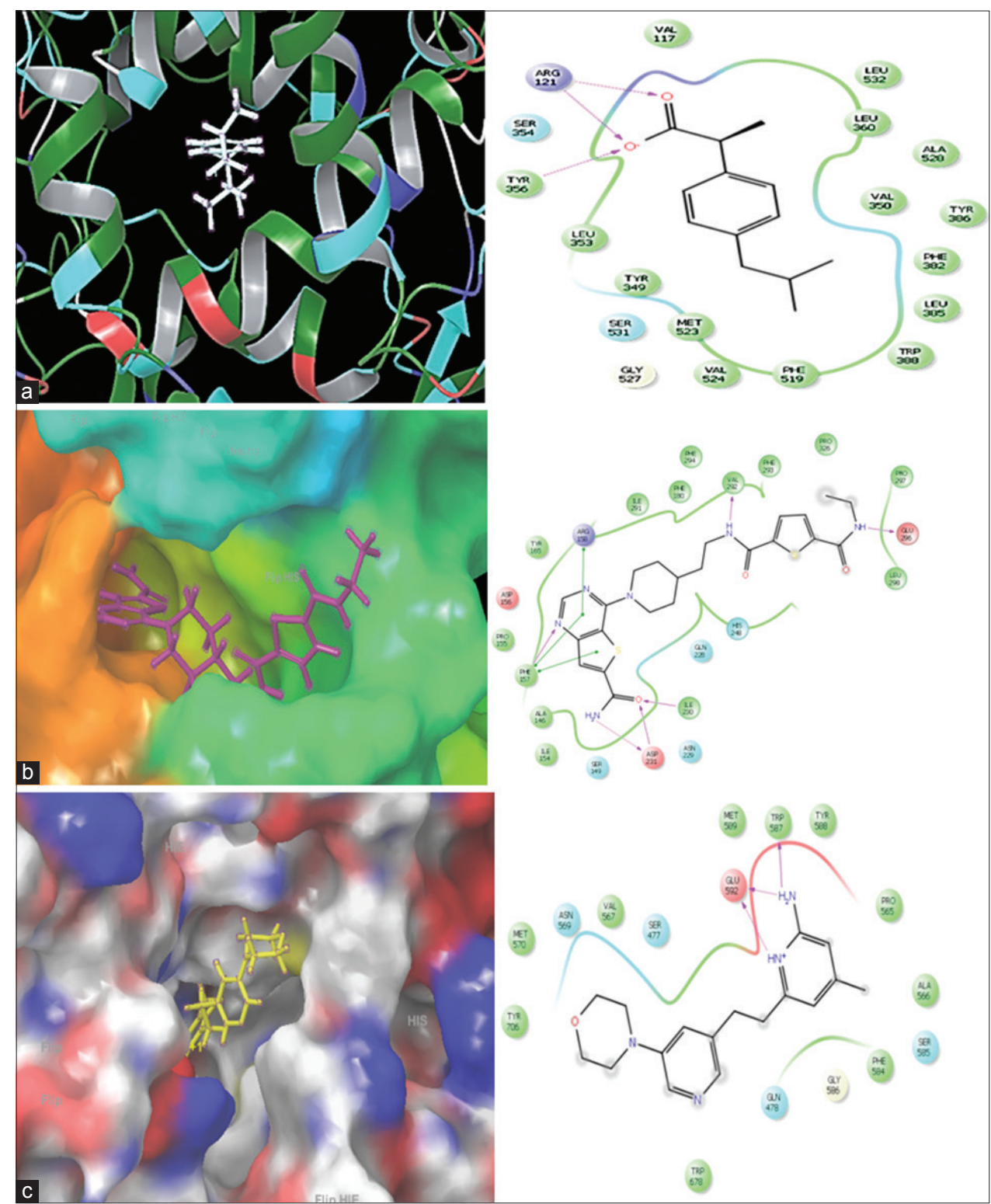

Fig. 2: The three crystal ligand in the active site of protein and its ligand interaction. (a) Ibuprofen ligand bound with protein Cox-2, (b) NAD ${ }^{+}$ligand bound with protein SIRUTIN, (c) inhibitor bound with nitric oxide synthase 
After reference ligand docking studies, the energy-based pharmacophore hypothesis was developed by mapping energetic terms onto pharmacophore sites. The results of the e-phamacophore generation of the reference ligands were used to identify the best pharmacophore hypothesis. The energy contribution for binding of a ligand to the protein was the key to derive pharmacophoric features in structure-based design. The diagrammatic representation for the energy-based pharmacophores generated was depicted in Fig. 4a-c, respectively.

The first generated e-pharmacophore showed 1 aromatic ring and 1 hydrophobe which is needed for the hydrophobic interaction within the pocket namely (R3) with its score $(-1.05)$ and (H1) its score (0.63) and one negative ionisable group (N2) with the score of $(-1.83)$. The second energy based pharmacophore hypothesis were developed and the features were found with two acceptors namely (A2 and A5) with the score ( -2.20 and -0.75$)$, two aromatic rings (R13 and R14) its score $(0.77$ and -1.45$)$ required for hydrophobic interaction, and 3 donors
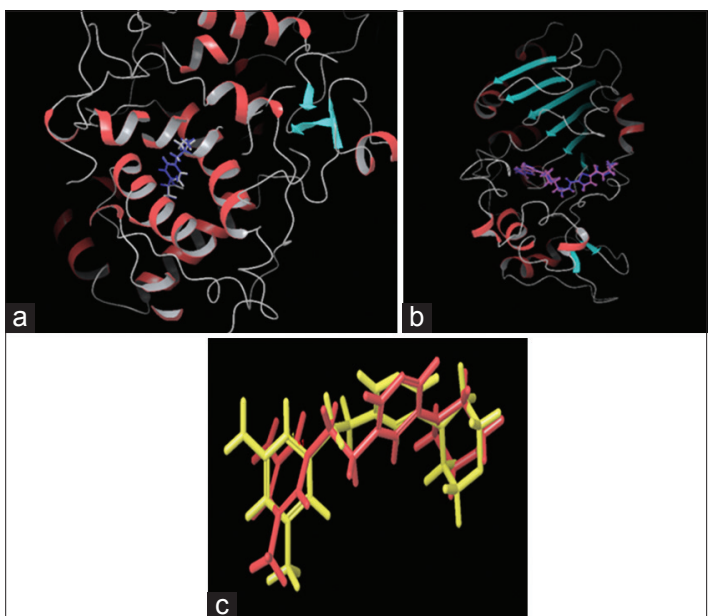

Fig. 3: The three superimposition of docked pose of ibuprofen, $\mathrm{NAD}^{+}$, Nitric oxide inhibitor and original pose of ibuprofen, $\mathrm{NAD}^{+}$and Nitric oxide Inhibitor; (where grey color indicates the original ligand and the violet color represents docked ligand), (where violet color indicates the original ligand and the pink color represents docked ligand), (where yellow color indicates the original ligand and the red color represents docked ligand). (a) Ibuprofen ligand with protein, (b) $\mathrm{NAD}^{+}$ligand with protein, (c) nitric oxide inhibitor
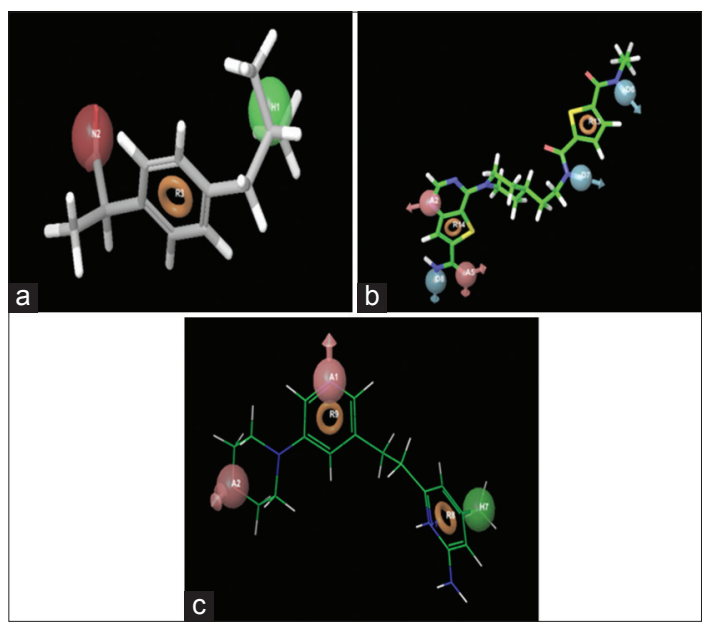

Fig. 4: The three energy-based pharmacophore generation for virtual screening of compounds. (a) Ibuprofen ligand with protein, (b) $\mathrm{NAD}^{+}$ligand with protein, (c) nitric oxide inhibitor
(D6, D7 and D8) and their corresponding score of $(-0.39,0.62$ and 0.35$)$ and the third energy based pharmacophore hypothesis were developed and the features were found1acceptor, namely, (A1) with score $(-0.67)$, two aromatic rings (R8 and R9) and their score ( -1.05 and -0.89$)$ 1 hydrophobic (H1) interaction and its score (0.25) and 1 donors (D2) and its score (0.67). The energy score of the pharmacophoric features for all crystal structure is represented in Table 1a-c, respectively.

Virtual screening of natural products was crucial on in-silico drug discovery process as it helps in the selection of novel bioactive compounds. We have selected 14 molecules for our study (Fig. 1). Three docking strategies were used for our study. Top hits from HTVS with the docking score of $-5.0 \mathrm{kcal} / \mathrm{mol}$ were subjected to secured screening using glide SP. Using XP docking analysis, it is very easy to predict hydrogen bond interactions, electrostatic interaction, hydrophobic interactions, and stacking interaction. Finally, XP docking studies were done for three targets and found that the most active compound after XP screening was cycloepoxytriol, phomol and phomol with docking score of $-7.511 \mathrm{kcal} / \mathrm{mol},-9.77 \mathrm{kcal} / \mathrm{mol}$ and $-9.97 \mathrm{kcal} / \mathrm{mol}$. Molecular docking studies were carried out, and the corresponding docking scores for all the targets were tabulated in Table 2a-c, respectively.

\section{DISCUSSIONS}

Developing novel endophytic bioactive compounds is one of the most promising drug targets for anti-inflammatory, anticancer, and antioxidant drug discovery. The screened 14 natural compounds were ranked based on the binding affinity, calculated as the scoring function called Glide score or docking score. The compounds were identified using phase find matches using phase module available in the Schrodinger Software.

Cyclooxygenase-2 enzyme was retrieved from protein data bank for anti-inflammatory drug screening. The reference ligand after docking was found to well fit into the active site of the enzyme cavity. The closer analysis of the molecule was found that the compound has two important interaction with Arg121 and Tyr356. Fuethermore, the ligand was entirely surrounded with hydrophobic amino acids Phe519, Trp388, Met523 and Leu353. On screening of 14 natural bioactive compounds, the most active compound cycloepoxytriol after XP

Table 1: Anti-inflammatory, anticancer and antioxidant energy based pharmacophore features and its scores

a. Energy-based pharmacophore generation for virtual screening of compounds (Ibuprofen bound with Cox-2)

\begin{tabular}{lll}
\hline Feature label & Score & Score source \\
\hline N2 & -1.83 & Negative ionisable \\
H1 & 0.63 & Hydrophobe \\
R3 & -1.05 & Aromatic ring \\
\hline
\end{tabular}

b. Energy-based pharmacophore generation for virtual screening of compounds (NAD ${ }^{+}$bound with SIRUTIN)

\begin{tabular}{lll}
\hline A5 & -0.75 & Hydrogen bond acceptor \\
D6 & -0.39 & Hydrogen bond donor \\
D7 & -0.62 & Hydrogen bond donor \\
D8 & -0.35 & Hydrogen bond donor \\
A2 & -2.2 & Acceptor \\
R13 & -0.77 & Ring group \\
R14 & -1.45 & Ring group \\
\hline
\end{tabular}

c. Energy-based pharmacophore generation for virtual screening of compounds (nitric oxide inhibitor bound with nitric oxide synthase)

\begin{tabular}{lll}
\hline A1 & -0.67 & Hydrogen bond acceptor \\
A2 & -0.67 & Hydrogen bond donor \\
H7 & -0.25 & Hydrophobe \\
R9 & -0.89 & Ring group \\
R8 & -1.05 & Ring group \\
\hline
\end{tabular}


docking analysis was found to be $-7.511 \mathrm{kcal} / \mathrm{mol}$. The binding analysis of the protein and ligand was depicted in Fig. $5 \mathrm{a}$. On closer analysis, the ligand was found to interact with an amino acid Tyr356 which is similar to the reference ligand and also the hydroxyl moiety was surrounded by hydrophobic amino acids like Phe519, Val524 and Met523. As this molecule was found to well buried into the active site pocket which makes this molecule a top active molecule than others [17].

SIRUTIN-3 enzyme was taken for anticancer study as SIRT-3 gene is over-expressed in most of the cancers such as lung and prostrate. A similar way of the study was performed for this target too $[12,13]$. The reference ligand $\mathrm{NAD}^{+}$was found to interact with positively charged amino acid like Glu296, Arg158, and Asp231. After three different docking analysis, the most active compound Phomol was found to be interact with amino acids Phe157 and Tyr165 with the binding score of $-9.77 \mathrm{kcal} / \mathrm{mol}$. The three dimensional view of the ligand and the

Table 2: Docking analysis and their scores for the top leads

\begin{tabular}{llll}
\hline Compounds & $\begin{array}{l}\text { HTVS } \\
\text { kcal/mol }\end{array}$ & SP kcal/mol & XP kcal/mol \\
\hline $\begin{array}{l}\text { a. Anti-inflammatory } \\
\text { target }\end{array}$ & & & \\
Cycloepoxytriol & -5.673 & -7.354 & -7.511 \\
Cycloepoxylactone & -4.977 & -7.148 & -7.428 \\
Phyllostine & -6.283 & -6.800 & -6.429 \\
Phomoxanthone A & -6.065 & -6.645 & -5.356 \\
3-Nitropropionic acid & -4.413 & -5.524 & -5.992 \\
Epioxydon & -5.722 & -5.790 & -4.901 \\
b. Anticancer target & & & \\
Phomol & -6.547 & -7.247 & -9.778 \\
Phomodione & -6759 & -8.362 & -9.260 \\
Phomoxanthone & -7.610 & -7.763 & -7.092 \\
Cycloepoxylactone & -6.441 & -6.491 & -6.991 \\
Phomosine & -5.731 & -6.593 & -6.758 \\
Epioxydon & -5.075 & -7.098 & -6.375 \\
Cycloepoxytriol & -6.290 & -6.290 & -5.670 \\
3-nitropropionic acid & -3.564 & -4.349 & -2.637 \\
c. Anti-oxidant target & & & \\
Phomol & -5.399 & -7.021 & -9.970 \\
Monodeacetyl & -7.021 & -6.959 & -8.528 \\
phomoxanthone B & & & \\
Phomoxanthone A & -3.943 & -5.194 & -7.795 \\
Cycloepoxylactone & -4.193 & -5.214 & -6.646 \\
Trihydroxy-p-methane & -5.064 & -5.279 & -5.840 \\
Phyllostine & -4.560 & -5.279 & -5.561 \\
Cycloepoxytriol B & -4.035 & -4.366 & -5.327 \\
Phomodione & -4.824 & -4.837 & -5.285 \\
Epioxydon & -4.453 & -4.969 & -3.496 \\
3-Nitropropionic acid & -4.343 & -5.103 & -3.177 \\
Dicerandrol A & -2.524 & -3.438 & -0.166 \\
\hline
\end{tabular}

HTVS: High-throughput virtual screening protein reveals that the most active compound was well buried into the active site pocket of the protein which makes this molecule a better target than other compounds which were screened for the docking analysis. The binding analysis and the ligand interaction of the protein with ligand were shown in Fig. $5 b$.

Nitric oxide synthase enzyme crystal structure complexed with inhibitor was taken for our study to discover novel hit molecule for antioxidant drug discovery. The reference ligand was docked into the active site of the enzyme, and the docking score was found to be $-6.637 \mathrm{kcal} / \mathrm{mol}$. The reference ligand was found to interact with positively charged amino acid Asn569 and stacking interaction with Glu592. After three different docking strategies, the compound Phomol was found to be $-9.970 \mathrm{kcal} / \mathrm{mol}$. The closer analysis of the compound was analyzed and found that the compound was found to interact with the amino acids Glu592 and Trp587 also the propyl group of this compound was found to interact with non-polar amino acid Ala475, Phe584, Ile459 and Leu424 amino acids. The binding analysis and the ligand interaction diagram for the most active compound were shown in Fig. 5c.

To further account for the potential of the compounds to act as efficient drug candidates, their ADME properties were also predicted using quickprop module in Schrodinger. The obtained values for molecular $\log$, HERG property, Caco accessibility, blood-brain barrier, and human oral absorption; it is also used to access violation of Lipinski's rule of five for the prompt drug candidate. All the compounds were shown to correlate well with the human oral absorption; blood-brain barrier separates the human brain from the direct contact of circulatory system, thus protecting the brain for unwanted solute particles. Both the most active compounds shown to be blood brain barrier negative ensuring their administration safe for the brain. Furthermore, all the compounds followed Lipinski's rule of five. The ADME prediction for all the compounds was reported in Table 3.

\section{CONCLUSION}

In conclusion, using structure-based drug design, we have obtained some promising leads for anti-inflammatory, anticancer, and antioxidant drug discovery. The discovery of compounds from natural products is very potent for formulating new drugs. Endophytic fungi are producing more bioactive compounds analogs similar to their hosts. Based on our analysis, we can say that the endophytic fungi could be a reliable source for pharmaceutically and industrially important compounds for drug discovery and development. Based on our study, we have found that the most active anti-inflammatory compound was found to be cycloepoxytriol with the docking score of $-7.511 \mathrm{kcal} / \mathrm{mole}$ and the binding analysis of the compound was found to be that the compound was well buried into the active of the protein which implies the high activity of the compound make this molecule more potent. The most active anticancer compound was found to be Phenol with the docking score of $-9.778 \mathrm{kcal} / \mathrm{mole}$ and the binding analysis of the compound

Table 3: ADME prediction for the natural endophytic bioactive compounds

\begin{tabular}{lllllll}
\hline Compound ID & QPlogPo/w $^{\mathrm{a}}$ & QPlogHERG $^{\mathrm{b}}$ & QPPCaco $^{\mathbf{c}}$ & QPlogBB $^{\mathrm{d}}$ & \% Human oral absorption $^{\mathrm{e}}$ & Rule of five $^{\mathbf{f}}$ \\
\hline Cycloepoxylactone & 0.109 & -3.007 & 462.605 & -0.808 & 75.288 & 0 \\
Cycloepoxytriol & -0.269 & -2.549 & 653.53 & -0.681 & 75.76 & 0 \\
3-Nitropropionic acid & -0.542 & -0.83 & 25.898 & -1.226 & 49.065 & 0 \\
Dicerandrol & 3.87 & -5.88 & 6.588 & -3.993 & 38.344 & 2 \\
Phomodione & 1.09 & -3.469 & 338.281 & -1.129 & 78.599 & 0 \\
Phomol & 2.844 & -4.882 & 223.94 & -1.918 & 85.658 & 0 \\
Phomosine G & 3.492 & -4.202 & 675.49 & -0.725 & 100 & 2 \\
Phomoxanthone & 2.953 & -4.456 & 15.852 & -2.779 & 39.801 & 0 \\
Phyllostine & -1.966 & -1.122 & 307.145 & -0.556 & 59.951 & 0 \\
Trihydroxy-p-menthane & 1.348 & -2.817 & 1464.461 & -0.366 & 91.501 & \\
\hline
\end{tabular}

aPredicted octanol/water partition coefficient $\log$ P (acceptable range: $-2.0-6.5$ ); ${ }^{\text {PPredicted IC }}$, value for blockage of HERG K+channels (below -5); cPredicted apparent

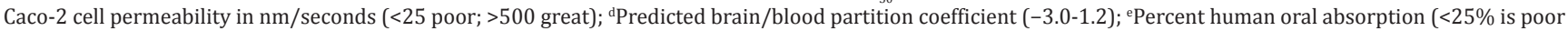

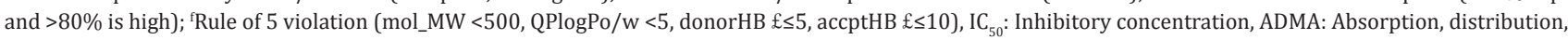
metabolism and excretion 

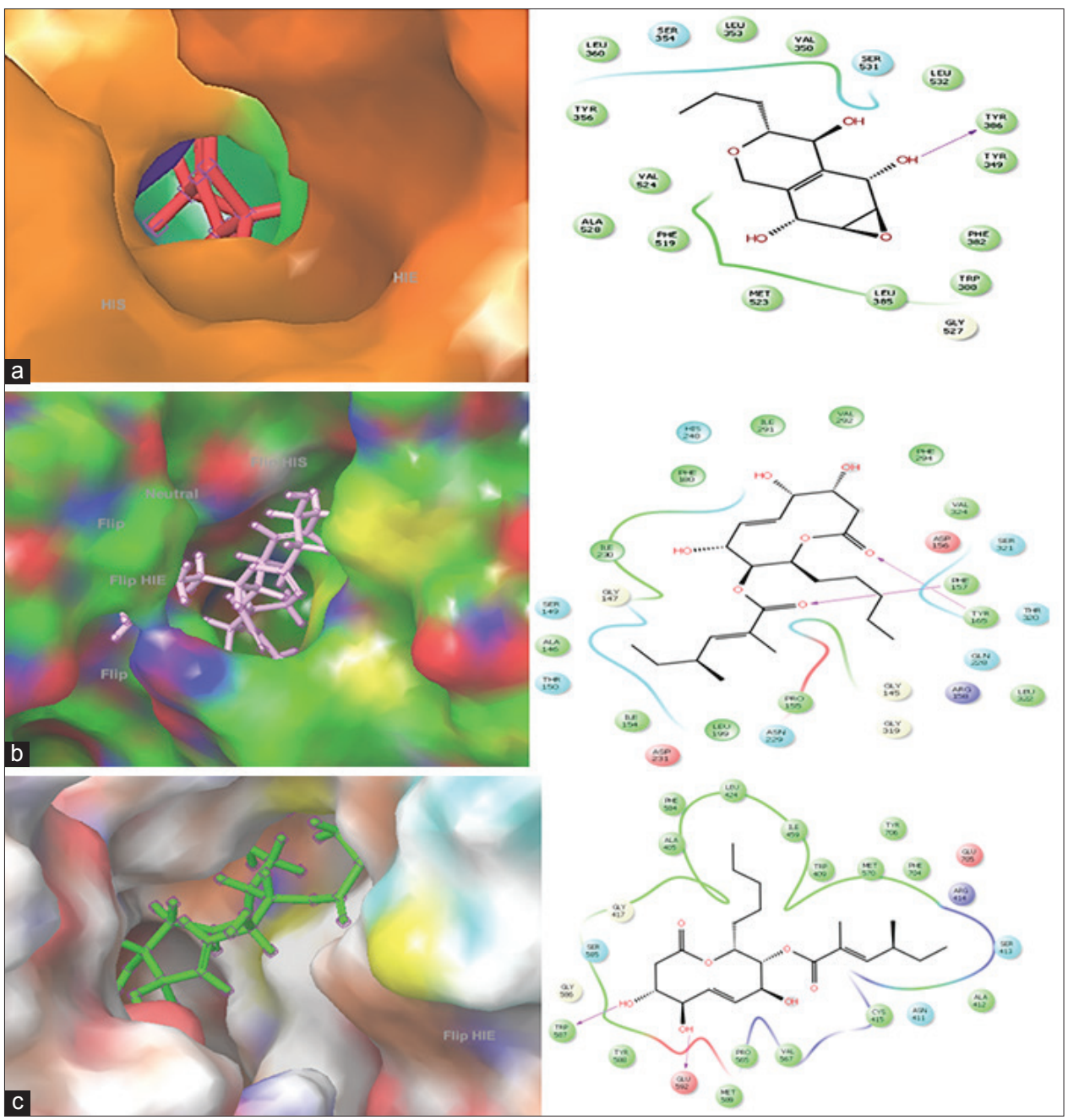

Fig. 5: (a) Binding analysis for the compound cycloepoxytriol with Cox-2 protein, (b) binding analysis for the compound phomol with SIRUTIN protein, (c) binding analysis for the compound phomol with nitric oxide synthase

found to be interact with the amino acid interaction Phe157 and Tyr165. The most active antioxidant compound was found to be Phenol with the docking score of $-9.970 \mathrm{kcal} / \mathrm{mole}$ and the binding analysis was found to be interacting with Trp587 and Glu592. The molecule was buried with the hydrophobic pocket which shows the activity of the molecule. To conclude, the natural endophyte Phomol was found to be most active according to structure-based drug design strategy. The most active bioactive compounds did not show any violation in ADME predication using quickprop as a module in Schrodinger. Further the top active compounds after virtual screening will be carried out for their in-vitro analysis, QSAR analysis [18] and to prove the better target for anti-inflammatory, anticancer, and antioxidant targets.

\section{ACKNOWLEDGMENT}

We authors thank Dr. M. Chandrasekar, Director, Department of Mechanical Engineering, Vels University for providing us the Computer system with all the configuration which we needed for our study.

\section{REFERENCES}

1. Bacon CW, White JF. Microbial Endophytes. New York: Marcel Dekker; 2000. p. 341-88.

2. Deshmukh SK, Mishra PD, Kulkarni-Almeida A, Verekar S, Sahoo MR, Periyasamy G, et al. Anti-inflammatory and anticancer activity of ergoflavin isolated from an endophytic fungus. Chem Biodivers 2009;6(5):784-9.

3. Strobel G, Ford E, Worapong J, Harper JK, Arif AM, Grant DM, et al. Isopestacin, an isobenzofuranone from Pestalotiopsis microspora, possessing antifungal and antioxidant activities. Phytochemistry 2002;60(2):179-83.

4. Saxena S, Devi PB, Soni V, Yogeeswari P, Sriram D. Identification of novel inhibitors against Mycobacterium tuberculosis L-alanine dehydrogenase (MTB-AlaDH) through structure-based virtual screening. J Mol Graph Model 2014;47:37-43.

5. Salam NK, Nuti R, Sherman W. Novel method for generating structurebased pharmacophores using energetic analysis. J Chem Inf Model 2009;49(10):2356-68.

6. Nagamani SC, Erez A, Lee B. Argininosuccinate lyase deficiency. Genet Med 2012;14(5):501-7.

7. Friesner RA, Banks JL, Murphy RB, Halgren TA, Klicic JJ, Mainz DT, et al. Glide: A new approach for rapid, accurate docking and scoring 1. Method and assessment of docking accuracy. J Med Chem 2004;47(7):1739-49.

8. Kawatkar S, Wang H, Czerminski R, Joseph-McCarthy D. Virtual fragment screening: An exploration of various docking and scoring protocols for fragments using Glide. J Comput Aided Mol Des 2009;23(8):527-39.

9. Alverez J, Shoichet B. Virtual Screening in Drug Discovery. Boca Raton, Florida: Taylor Francis; 2005.

10. Duggan KC, Hermanson DJ, Musee J, Prusakiewicz JJ, Scheib JL, Carter $\mathrm{BD}$, et al. (R)-profens are substrate-selective inhibitors of endocannabinoid oxygenation by COX-2. Nat Chem Biol 2011;7(11):803-9.

11. Smith WL, Urade Y, Jakobsson PJ. Enzymes of the cyclooxygenase pathways of prostanoid biosynthesis. Chem Rev 2011;111(10):5821-65.

12. Deng CX. SIRT1, is it a tumor promoter or tumor suppressor? Int J Biol Sci 2009;5(2):147-52.

13. Finkel T, Deng CX, Mostoslavsky R. Recent progress in the biology and physiology of sirtuins. Nature 2009;460(7255):587-91. 
14. Allison SJ, Milner J. SIRT3 is pro-apoptotic and participates in distinct basal apoptotic pathways. Cell Cycle 2007;6(21):2669-77.

15. Smyth EM, Grosser T, Wang M, Yu Y, FitzGerald GA. Prostanoids in health and disease. J Lipid Res 2009;50 Suppl: S423-8.

16. Valko M, Leibfritz D, Moncol J, Cronin MT, Mazur M, Telser J. Free radicals and antioxidants in normal physiological functions and human disease. Int J Biochem Cell Biol 2007;39(1):44-84.
17. Naresh K, Ramakrishnan G. Molecular docking studies of designed benzamide derivatives as histone deacetylase 2 inhibitors. Int J Pharm Pharm Sci 2014;6(4):324-8.

18. Rajamanikandan S, Sindhu T, Durgapriya D, Anitha JR, Akila S, Gopalakrishnanet VK, et al. Molecular docking and QSAR studies on bioactive compounds isolated from marine organisms into the MUC1 oncoprotein. Int J Pharm Pharm Sci 2011;3:168-72. 